\title{
Application of endobronchial ultrasound-guided transbronchial needle aspiration in mediastinal lymphangioma
}

\author{
Runbo Zhong ${ }^{1 \#}$, Xiaoxuan Zheng ${ }^{1,2 \#}$, Jiajun Teng ${ }^{1}$, Baohui Han ${ }^{1}$ \\ ${ }^{1}$ Department of Pulmonary Medicine, ${ }^{2}$ Department of Endoscopy, Shanghai Chest Hospital, Shanghai Jiao Tong University, Shanghai 200030, China \\ Contributions: (I) Conception and design: B Han; (II) Administrative support: J Teng; (III) Provision of study materials or patients: J Teng, X Zheng, \\ R Zhong; (IV) Collection and assembly of data: R Zhong; (V) Data analysis and interpretation: R Zhong; (VI) Manuscript writing: All authors; (VII) \\ Final approval of manuscript: All authors. \\ "These authors contributed equally to this work. \\ Correspondence to: Baohui Han. Department of Pulmonary Medicine, Shanghai Chest Hospital, Shanghai Jiao Tong University, 241 West Huaihai \\ Road, Shanghai 200030, China. Email: hanxkyy@aliyun.com.
}

\begin{abstract}
Background: Mediastinal lymphangioma is a rare lymphatic malformation, and the standard treatment strategy is surgical dissection. Endobronchial ultrasound-guided transbronchial needle aspiration has good diagnostic abilities for paratracheal, mediastinal, and hilar lymph node lesions. Endoscopic ultrasound is a new technique which can be used for the treatment of mediastinal lymphangioma to reduce the incidence of surgical-related complications. This study was designed to investigate the value of endobronchial ultrasoundguided transbronchial needle aspiration in the treatment of mediastinal lymphangioma.

Methods: Retrospective analysis was carried out on nine patients with mediastinal lymphangioma who underwent endoscopic ultrasound-guided fine-needle aspiration from 2010 to 2018 in Shanghai Chest Hospital.

Results: No patients suffered serious complication. The amount of fluid aspirated was 50-205 mL. The disease was stable over a period of 9 months to 2 years.

Conclusions: Endobronchial ultrasound-guided transbronchial needle aspiration could be an effective method for the treatment of mediastinal lymphangioma with a little trauma compared with surgical dissection, which may have significant therapeutic effects.
\end{abstract}

Keywords: Mediastinal lymphangioma; endoscopic ultrasound (EBUS); transbronchial needle aspiration (TBNA)

Submitted May 03, 2019. Accepted for publication Aug 08, 2019.

doi: $10.21037 /$ atm.2019.08.71

View this article at: http://dx.doi.org/10.21037/atm.2019.08.71

\section{Introduction}

Lymphangioma is a rare lymphatic malformation that is usually considered to be caused by a congenital lymphatic developmental malformation or by disease or traumatic injury to the lymphatic vessels. It is a benign tumor formed by abnormally proliferating and dilated lymphatic vessels. Lymphangiomas are common in infants' head and neck, and less than $1 \%$ occur in the mediastinum $(1,2)$. Adult mediastinal lymphangioma is even rarer in clinical practice, accounting for $0.7 \%$ to $4.5 \%$ of mediastinal tumors $(3,4)$. Longitudinal lymphangioma is usually asymptomatic and can compress the surrounding organs due to local enlargement, resulting in compression symptoms.

Surgical resection is generally considered the standard treatment for mediastinal lymphangioma; however, the chances of surgical trauma and related complications are high. Ultrasound bronchoscopy is a method for adding an ultrasound probe at the front end of the bronchoscope. It can be used for real-time ultrasound-guided transbronchial fine-needle aspiration and is mainly used for the diagnosis of unexplained hilar, mediastinal lymphadenopathy, and tracheal and bronchial masses. Because ultrasound bronchoscopy can be used to determine the location and density of lesions around the trachea and bronchi by EBUS, 
the entire contents of the lesion can be completely aspirated by changing the depth and position of the needle, which could be used for mediastinal lymphangioma treatment. Nevertheless, there is little information on its use in patients with mediastinal lymphangioma. Herein, we report the use of endobronchial ultrasound-guided transbronchial needle aspiration (EBUS-TBNA) in nine patients with mediastinal lymphangioma from 2010 to 2018, using a retrospective study analysis. Meanwhile, there were ten patients during this 8-year period underwent surgical resections diagnosed with mediastinal lymphangiomas. And unfortunately, 1 of the 10 patients suffered from chylopleura after the surgery.

\section{Methods}

\section{Clinical demography}

A total of nine patients with clinic-radiological mediastinal lymphangioma underwent EBUS-TBNA scans from October 2010 to December 2018. There were four males and five females, aged 42-83 years (median age of 55 years). In the physical examination, three patients were asymptomatic, and 6 of the 9 patients had the following symptoms: two patients had cough, two patients had shortness of breath, one patient had a hoarse voice, and one patient had a fever. Two patients had a smoking history. The preoperative longest diameter of lymphangioma, using a computed tomography (CT) scan, was $2-6 \mathrm{~cm}$. Eight cases of lymphangioma were located in the posterior superior vena cava of the right superior mediastinum, and one was multiple lymphangioma, located under the aortic arch, adjacent to the aortic arch and left hilar. None of the patients had any primary lung disease. The clinical data of the patients are shown in Table 1.

\section{Inclusion criteria}

(I) All patients were diagnosed as mediastinal lymphangioma by chest-enhanced CT scans before operation, and the image data were analyzed and confirmed by two experienced radiologists;

(II) The longest diameter of mediastinal lymphangioma was $>1 \mathrm{~cm}$;

(III) Cardiopulmonary function was normal, and there was no obvious damage to the liver, kidney, or the hematopoietic system;

(IV) A signed informed consent form was obtained from each patient.

\section{EBUS-TBNA procedure}

All patients underwent EBUS-TBNA under local anesthesia, abstaining from solid food and liquids for at least $4 \mathrm{~h}$ before the procedure. After achieving vascular access for intravenous infusions, local anesthesia was achieved with $2 \mathrm{~mL} 2 \%$ lidocaine solution, orally, plus 3-5 sprays of $7 \%$ lidocaine solution to the pharynx. The regular bronchoscopy was performed first, and all samples were subjected to fluorescence scanning. After oral bronchoscopy, target lesions and peripheral vessels were examined by EBUS, using a linear array ultrasonic bronchoscope (BF-UC 260F-OL8 or BF-UC260FW; Olympus Ltd., Tokyo, Japan). Scanning was performed at a frequency of $7.5 \mathrm{MHz}$ and images were processed by an Olympus ultrasound processor (EU-C2000 or EU-ME1; Olympus Ltd.). Target lesion diameter was measured and recorded under frozen ultrasound image. A dedicated 22-gauge needle was used (NA-201SX-4022; Olympus Ltd.) to perform aspiration under real-time ultrasound guidance, confirming that the aspiration needle reached the target area for specimen withdrawal. The Doppler function was enabled before aspiration to prevent the needle from penetrating into the blood vessel. The cytology smears of the aspiration specimens were examined by the same experienced pathologists. Microbiological tests were performed as deemed appropriate.

\section{Results}

\section{Side effects}

None of the nine patients showed any significant adverse effect during or after treatment with EBUS-TBNA. During the procedure, no serious complication, including pneumothorax, mediastinal emphysema, or mediastinal rupture of the mediastinum, was observed except for a slight bleeding in the aspiration spot as observed by the endoscope.

\section{Clinical results}

Of the nine patients, three patients had local extrinsic compression on the right tracheal mucosa as observed in a regular bronchoscopy procedure, and the other six patients had no abnormality from regular bronchoscopy. All nine patients showed no abnormality in the fluorescence bronchoscopy. The number of aspiration times required per 


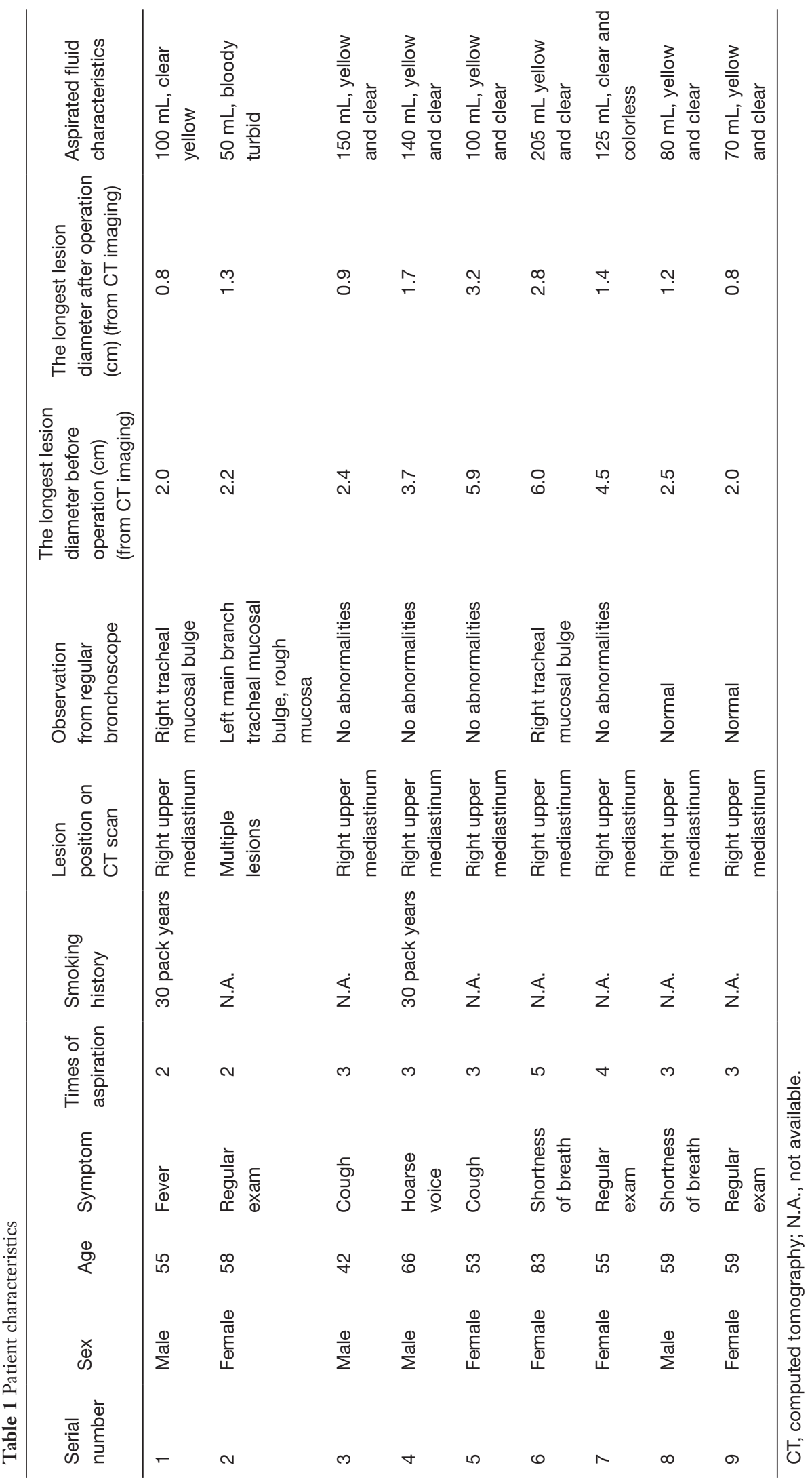



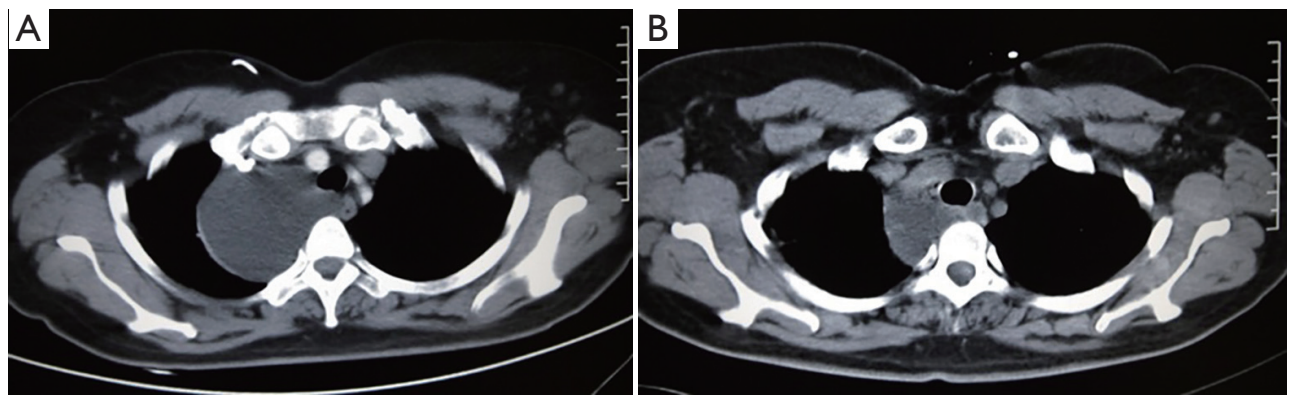

Figure 1 CT images before (A) and after (B) treatment. CT, computed tomography.

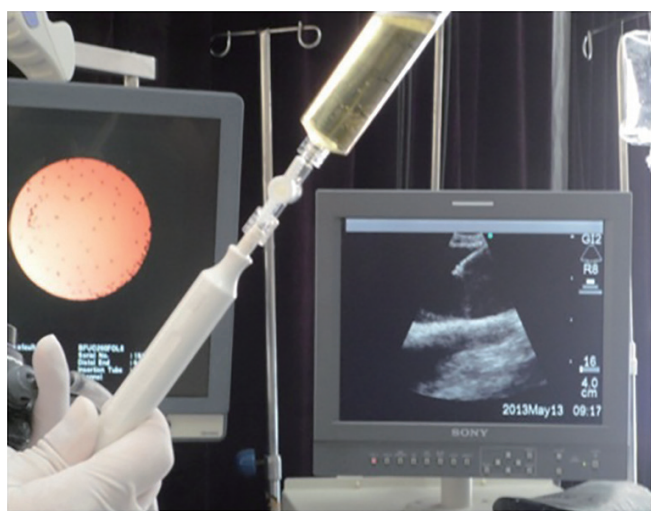

Figure 2 Ultrasound images.

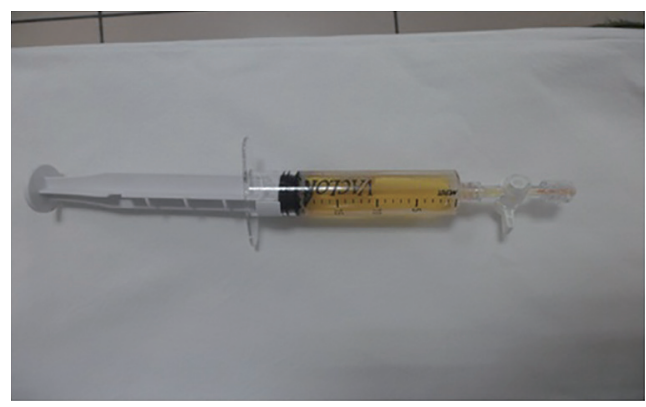

Figure 3 Aspiration fluid.

patient for lymphangioma under endoscopic ultrasound was 2-5 (median: 3), while the number of passes depending on liquid volume and scope. The volume of liquid withdrawn was 50-205 mL. In seven cases, the liquid was colored yellow, whereas one patient had colorless and clear liquid, and in one patient the liquid had a bloody turbidity. Under the microscope, mature lymphocytes were dominant in the aspiration smear. The results of bronchoscopy and aspiration of all nine patients are summarized in Table 1 .

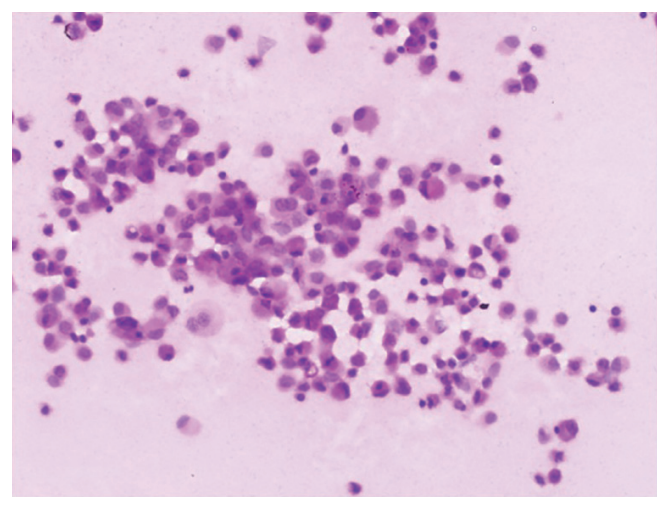

Figure 4 Pathological results (HE, ×400, mature lymphocytes were dominant in the aspiration smear).

In the follow-up period, nine patients had a stable disease (the longest diameter of mediastinal lymphangioma did not increase by more than $50 \%$ of the diameter after the operation) for more than 9 months, and a 53 -year-old female patient was found to have an enlarged lymphangioma lesion similar to the original size after treatment at 9 months of follow-up. That patient was subject to TBNA aspiration again, and found to have $105 \mathrm{~mL}$ of yellow, clear liquid, and was followed-up with a local injection of 500,000 units of interleukin-2 (IL-2). The longest period of disease stabilization in the nine patients was 2 years, and this patient is still being followed-up.

The CT images, ultrasound images, aspiration fluid, and pathological results from a representative patient before and after treatment are shown in Figures 1-4.

\section{Discussion}

Lymphangioma is a relatively rare benign tumor that occurs in the lymphatic system, and that could occur in any area of the body containing lymphatic vessels. About $90 \%$ of 
lymphangiomas are observed in children under 2 years of age, and are more commonly encountered in the neck and armpits (4-6). Primary mediastinal lymphangioma is rare, accounting for about $1 \%$ of systemic lymphangioma, with almost all cases occurring in adults and are more common in women (3,7-9). The majority of mediastinal lymphangioma are located in the anterior superior mediastinum, with the right side more than the left side. The anatomical basis for the anterior superior mediastinum location is still unclear.

Lymphangioma is located in the neck or armpits of children, often accompanied by involvement of tissue components, and could be regarded as a congenital disease, whereas adult lymphangioma is more common in the mediastinum with a pure liquid in the cyst, suggesting that it may be a secondary disease. Congenital lymphangioma is a lymphatic vessel disease that is not connected to veins or other lymphatic vessels during lymphatic development. The drainage is blocked, and the lymphatic lumen gradually expands to form lymphangioma. Secondary lymphangioma is often caused by blockage of lymphatic vessels by certain diseases or a possible lymphatic injury (3). In recent years, there have been reports that lymphangioma might be attributed to a possible neoplastic nature related to the PIK3CA mutation (10). The average age of the nine patients in the present study was 55 , and all denied a history of trauma. Thus, they were clinically diagnosed as secondary lymphangioma patients. Lymphangiomas are classified into three types based on their tissue structures: capillary lymphangioma, cystic lymphangioma, and cavernous lymphangioma. Among them, cystic lymphangioma is the most common type $(4,11,12)$.

Although mediastinal lymphangioma is benign, it has the characteristics of invasive growth in the body. With the enlargement of the lesion and the increase in internal tension, the surrounding organs could be compressed, and local bleeding or infection may occur. The patient may have no symptom for years until coughing, dyspnea, and dysphagia occur when the vital organs, including the trachea, esophagus, and great vessels, are seriously compressed (2). When the lymphangioma is complicated with secondary infection or intracapsular hemorrhage, the lesion volume could increase markedly. Compression of surrounding tissues leads to serious consequences, including dysphagia and dyspnea. A low proportion of patients may have complications including a chylothorax (13). Therefore, early diagnosis and active treatment are necessary.

Typical mediastinal lymphangioma in CT imaging presents as a uniform low-density fluid cystic mass, mostly round or lobulated, with clear boundaries. A few cases show unclear boundaries, spreading around the mediastinum or diffusing along the mediastinal macrovascular space. The enhanced CT scan mostly shows no enhancement. When a cystic mass is accompanied by blood or infection, it could result in an increased CT signal, which is higher than the surrounding soft tissue density. The magnetic resonance imaging (MRI) scans show that the T1-weighted image has a low signal, and the $\mathrm{T} 2$-weighted image has a high or equivalent signal $(3,14)$. MRI is generally considered to be superior to $\mathrm{CT}$ in the diagnosis of mediastinal lymphangioma. The nine patients in this study underwent only CT examination without MRI information, which is a limitation of this study. However, the CT scans showed uniform low-density liquid areas, and a large number of mature lymphocytes were observed on the specimen smears. The follow-up showed good effects, and the results could confirm the diagnosis of mediastinal lymphangioma. However, definitive histological diagnosis is difficult to achieve before surgery (2).

For the treatment of mediastinal lymphangioma, surgery is generally considered to be a standard treatment. The recurrence rate after complete lesion dissection is low. However, multiple dissections may be required for lymphangioma with extensive lesions $(15,16)$. Surgical trauma and complications are usually encountered, especially for aged patients. In this retrospective study from October 2010 to December 2018, we found there were another ten patients underwent surgical resections diagnosed with mediastinal lymphangiomas while nine had EBUS-TBNA. And unfortunately, 1 of the 10 patients who underwent surgical resection suffered from chylopleura after the surgery. With the development of minimally invasive techniques, several studies have focused on videoassisted thoracoscopic surgery (VATS) for the treatment of mediastinal cystic lymphangioma. However, VATS for treatment of mediastinal lymphangioma remains technically problematic because of the peripheral adhesions of the tumor $(6,17)$. So EBUS-TBNA should be the first choice or at least one of the optional treatments which could be used in patients of high surgical risks.

Ultrasound probes at the front end of the EBUS-TBNA bronchoscopy could be used to analyze the lesions around the trachea and bronchi, and the entire lesion could be completely eliminated by the changes of depth and position of the aspiration needle, which could be used as one of the treatment methods for mediastinal lymphangioma. In our retrospective analysis, patients who received EBUS- 
TBNA had a disease relief time of more than half a year, with the oldest patient being 83 years of age, indicating high clinical applicability. In addition, the injection of medicine into a lymphangioma under ultrasound guidance can prolong the control of lymphangioma (18). OK-432 is an immunomodulator, an inactive strain of group A Streptococcus pyogenes, that stimulates the lymphatic endothelial cell to produce an inflammatory response and hyperplasia of fibrous tissue for the occlusion of lymphatic vessels to achieve a reduction in lesion size. OK-432 has shown promising results in the treatment of large, unilocular cystic lymphangioma $(18,19)$. However, its efficacy in treating small cysts is lower (13). Shimizu et al. emphasized that with the injection of OK-432, temporary swelling and pyrexia may occur (20). Likewise, bleomycin is a poor candidate as it causes pulmonary fibrosis and has resulted in many adverse drug events, as reported in several studies. Zhou et al. found percutaneous drainage of a large mediastinal lymphangioma following fibrin glue injection to seal the cavity, allowing resolution of the patient's symptoms, with disappearance of the lesion and no clinical recurrence for up to 4 years (21).

In this study, we injected 500,000 units of IL-2 into one patient with relapsed lymphangioma, and the lesions did not increase in the short term. However, the lesion control time remains to be further observed.

In conclusion, EBUS-TBNA for the treatment of mediastinal lymphangioma showed a little trauma and significant therapeutic effects, and is an effective method for mediastinal lymphangioma management. The treatment is likely to be questioned in terms of prognosis and recurrence rate, but there is no doubt that it provides an alternative procedure to ease the pain of a patient who could not undergo the risk of surgery.

\section{Acknowledgments}

We would like to thank all of the investigators for their involvement in this study.

\section{Footnote}

Conflicts of Interest: The authors have no conflicts of interest to declare.

Ethical Statement: The authors are accountable for all aspects of the work in ensuring that questions related to the accuracy or integrity of any part of the work are appropriately investigated and resolved. The study protocol was approved by the Ethics Committee of Shanghai Chest Hospital (No. KS1933) and was conducted in accordance with the Helsinki Declaration of 1964 (revised 2008).

\section{References}

1. Brown LR, Reiman HM, Rosenow EC 3rd, et al. Intrathoracic lymphangioma. Mayo Clin Proc 1986;61:882-92.

2. Kanzaki M, Kikkawa T, Obara T, et al. Successful excision of an isolated mediastinal cystic lymphangioma with bilateral thoracoscopic surgery. Ann Thorac Cardiovasc Surg 2011;17:570-2.

3. Shaffer K, Rosado-de-Christenson ML, Patz EF Jr, et al. Thoracic lymphangioma in adults: CT and MR imaging features. AJR Am J Roentgenol 1994;162:283-9.

4. Faul JL, Berry GJ, Colby TV, et al. Thoracic lymphangiomas, lymphangiectasis, lymphangiomatosis, and lymphatic dysplasia syndrome. Am J Respir Crit Care Med 2000;161:1037-46.

5. Saleiro S, Magalhaes A, Moura CS, et al. Mediastinal cystic lymphangioma. Rev Port Pneumol 2006;12:731-5.

6. Hunt I, Eaton D, Dalal P, et al. Minimally invasive excision of a mediastinal cystic lymphangioma. Can J Surg 2009;52:E201-2.

7. Naidu SI, McCalla MR. Lymphatic malformations of the head and neck in adults: a case report and review of the literature. Ann Otol Rhinol Laryngol 2004;113:218-22.

8. Wilson C, Askin FB, Heitmiller RF. Solitary pulmonary lymphangioma. Ann Thorac Surg 2001;71:1337-8.

9. Yamagishi S, Koizumi K, Hirata T, et al. Experience of thoracoscopic extirpation of intrapulmonary lymphangioma. Jpn J Thorac Cardiovasc Surg 2005;53:313-6.

10. Luks VL, Kamitaki N, Vivero MP, et al. Lymphatic and other vascular malformative/overgrowth disorders are caused by somatic mutations in PIK3CA. J Pediatr 2015;166:1048-54.e1-5.

11. Oshikiri T, Morikawa T, Jinushi E, et al. Five cases of the lymphangioma of the mediastinum in adult. Ann Thorac Cardiovasc Surg 2001;7:103-5.

12. Teramoto K, Suzumura Y. Mediastinal cavernous lymphangioma in an adult. Gen Thorac Cardiovasc Surg 2008;56:88-90.

13. Peters DA, Courtemanche DJ, Heran MK, et al. Treatment of cystic lymphatic vascular malformations with OK-432 sclerotherapy. Plast Reconstr Surg 2006;118:1441-6. 
14. Charruau L, Parrens M, Jougon J, et al. Mediastinal lymphangioma in adults: CT and MR imaging features. Eur Radiol 2000;10:1310-4.

15. Park JG, Aubry MC, Godfrey JA, et al. Mediastinal lymphangioma: Mayo Clinic experience of 25 cases. Mayo Clin Proc 2006;81:1197-203.

16. Ming YC, Wong KS, Wang CJ, et al. Successful management of a bilateral diaphragmatic lymphangioma. Pediatr Surg Int 2007;23:591-3.

17. Lazopoulos G, Pavlopoulos D, Kambitakis E, et al. Huge cystic lymphangioma of the mediastinum successfully treated with thoracoscopic surgery. Ann Thorac Surg 2014;98:2233.

Cite this article as: Zhong $\mathrm{R}$, Zheng X, Teng J, Han B. Application of endobronchial ultrasound-guided transbronchial needle aspiration in mediastinal lymphangioma. Ann Transl Med 2019;7(18):450. doi: 10.21037/atm.2019.08.71
18. Acevedo JL, Shah RK, Brietzke SE. Nonsurgical therapies for lymphangiomas: a systematic review. Otolaryngol Head Neck Surg 2008;138:418-24.

19. Yoo JC, Ahn Y, Lim YS, et al. OK-432 sclerotherapy in head and neck lymphangiomas: long-term follow-up result. Otolaryngol Head Neck Surg 2009;140:120-3.

20. Shimizu J, Taga T, Kishimoto T, et al. Airway obstruction caused by rapid enlargement of cervical lymphangioma in a five-month-old boy. Clin Case Rep 2016;4:896-8.

21. Zhou S, Dong S, Du J. Percutaneous therapy of a mediastinal lymphangioma with fibrin glue: case report with clinical success after 4 years. BMC Surg 2018;18:4. 